\title{
X Congreso lbérico de Agroingeniería

\section{Estudio de la actividad antifúngica in vitro de materiales compuestos basados en complejos de inclusión de polifenoles contra Phytophthora cinnamomi}

\author{
Petruta Mihaela Matei ${ }^{1}$, Laura Buzón-Durán ${ }^{2}$, Eduardo Pérez-Lebeña², Jesús Martín-Gil², \\ Beatrice Michaela Iacomi ${ }^{1}$, M. Carmen Ramos-Sánchez ${ }^{3}$, Pablo Martín-Ramos, ${ }^{4}{ }^{*}$
}

1 Department of Bioengineering of Horticultural and Viticultural Systems, University of Agricultural Sciences and Veterinary Medicine of Bucharest, Bulevardul Mărăști 59, București 011464, Rumania; petruta.matei@horticultura-bucuresti.ro (P.M.M.); b.iacomi@yahoo.fr (B.M.I.)

2 Departamento de Ingeniería Agroforestal, ETSIIAA, Universidad de Valladolid, Avenida de Madrid 44, 34004 Palencia, España; laura.buzon.duran@gmail.com (L.B.-D.); eplebena@gmail.com (E.P.-L); mgil@iaf.uva.es (J.M.-G.)

3 Servicio de Microbiología y Parasitología, Hospital Universitario Rio Hortega, SACYL, Calle Dulzaina, 2, 47012 Valladolid, España; mramoss@saludcastillayleon.es

4 Instituto Universitario de Investigación en Ciencias Ambientales de Aragón (IUCA), EPS, Universidad de Zaragoza, Carretera de Cuarte, s/n, 22071 Huesca, España; pmr@unizar.es

Resumen: El hongo Phytophthora cinnamomi es responsable de la podredumbre radical en una amplia gama de hospedantes, produciendo grandes pérdidas económicas y ecológicas a nivel mundial. En el caso de España, es responsable de enfermedades como la 'seca' de la encina y el alcornoque, o la 'tinta' del castaño. En este estudio se han investigado diferentes compuestos de inclusión de polifenoles-esteviósido dispersados en una solución hidroalcohólica de oligómeros de quitosano, con aplicación como complejos naturales bioactivos para reemplazar a los fungicidas sistémicos convencionales. Los polifenoles ensayados in vitro han sido curcumina, ácido ferúlico, ácido gálico y silimarina. Se han ensayado tres concentraciones (125, 250 y 500 $\mu \mathrm{g} \cdot \mathrm{mL}^{-1}$ ), con y sin nanopartículas de plata (AgNPs), y se han encontrado diferencias notables en la inhibición del crecimiento del micelio, con valores de $\mathrm{CE}_{50}$ y CE90 que han oscilado entre 171 y $373,6 \mu \mathrm{g} \cdot \mathrm{mL}^{-1}$, y entre 446,2 y $963,7 \mu \mathrm{g} \cdot \mathrm{mL}^{-1}$, respectivamente, observándose un rendimiento superior de los preparados a base de ácido ferúlico con AgNPs y de silimarina sin nAg. Los resultados obtenidos ponen en evidencia que la adición de AgNPs, pese a su actividad antimicrobiana, no siempre da lugar a sinergismos. En el caso de P. cinnamomi, se ha encontrado un comportamiento antagonístico inesperado para dos de los polifenoles (curcumina y silimarina), un comportamiento aditivo para el ácido ferúlico y un comportamiento sinergístico para el ácido gálico. Los preparados referidos pueden ser prometedores para aplicaciones de protección de cultivos y masas forestales contra P. cinnamomi.

Palabras clave: antifúngico; nanoplata; podredumbre radical; polifenoles; sinergismo 


\author{
X CONGRESO IBÉRICO DE AGROINGENIERÍA \\ X CONGRESSO IBÉRICO DE AGROENGENHARIA \\ 3 - 6 septiembre 2019, Huesca - España
}

\title{
1. Introducción
}

Phytophthora cinnamomi es un oomiceto que vive en el suelo nutriéndose gracias a materia en descomposición y que produce una fitopatología (micosis) que afecta a cientos de huéspedes, una condición llamada "podredumbre de la raíz" o "muerte regresiva". Este patógeno es una de las especies más invasivas del mundo y afecta a una variedad de familias de plantas: coníferas, pastos, helechos, plantas ornamentales y cultivos alimentarios como la piña o el aguacate [1]. Su expansión ha sido atribuida al fenómeno del Cambio Climático [2]. Entre los síntomas de infección por P. cinnamomi figuran: marchitamiento, disminución del tamaño del fruto, muerte de brotes jóvenes, clorosis de las hojas y cancros del tallo [3].

En el caso de España, P. cinnamomi es responsable de enfermedades como la 'seca' de la encina y el alcornoque, o la 'tinta' del castaño [4].

Actualmente, no existe un tratamiento que erradique la muerte regresiva por P. cinnamomi, incluyendo la inyección al tallo de fosfitos (que son elicitores que actúan de manera indirecta como fungicidas) [5] o la pulverización foliar con metalaxyl+mancozeb (fungicidas con actividad por contacto y sistémica).

Como alternativa a estos agentes convencionales de tratamiento existe la posibilidad de utilizar complejos naturales bioactivos ricos en polifenoles. En este estudio se han investigado diferentes compuestos de inclusión de polifenoles-esteviósido dispersados en una solución hidroalcohólica de oligómeros de quitosano. Los polifenoles ensayados in vitro han sido curcumina, ácido ferúlico, ácido gálico y silimarina, a diferentes concentraciones, con y sin nanopartículas de plata (AgNPs).

\section{Material y métodos}

\subsection{Reactivos}

La curcumina (CAS 458-37-7), el ácido ferúlico (CAS 1135-24-6), el ácido gálico (CAS 149-917) y la silimarina (MDL MFCD01776359) se adquirieron de Sigma-Aldrich (Darmstadt, Alemania). El esteviósido (CAS 57817-89-7) se adquirió de Wako (Osaka, Japón). Hangzhou Simit Chemical Technology Co. Ltd. (Hangzhou, China) suministró el quitosano de peso molecular medio (MMWC). Todos los reactivos se utilizaron sin purificación adicional.

Los oligómeros de quitosano se prepararon a partir del MMWC de acuerdo con el procedimiento propuesto por Sun, et al. [6]: primero se solubilizaron $10 \mathrm{~g}$ de MMWC en $500 \mathrm{~mL}$ de ácido acético $(1 \%)$ con agitación constante a $60{ }^{\circ} \mathrm{C} y$, una vez disueltos, se obtuvieron oligómeros de quitosano (con $\mathrm{MW}<2 \mathrm{kDa}$ ) por degradación oxidativa con $\mathrm{H}_{2} \mathrm{O}_{2}\left(0,3 \mathrm{~mol} \cdot \mathrm{L}^{-1}\right)$.

\subsection{Preparación y caracterización de los compuestos de inclusión de polifenoles y composites bioactivos}

Los procedimientos para la preparación de compuestos de inclusión de polifenoles, mediante separación en un sistema bifásico acuoso asistida por microondas, y para la preparación de los compuestos con oligómeros de quitosano en solución hidroalcohólica fueron los mismos que los referidos previamente en Matei, et al. [7], asegurando así la comparabilidad de los resultados.

Para el medio basado en oligómeros de quitosano dispersos en solución hidroalcohólica, se agregaron $10 \mathrm{mg}$ de oligómeros de quitosano de $2 \mathrm{kDa}, 60 \mathrm{mg}$ de esteviósido y $10 \mathrm{mg}$ de uno de los polifenoles (curcumina, ácido férulico, ácido gálico o silimarina) a $40 \mathrm{~mL}$ de solución hidroalcohólica (1:1 v/v de agua destilada y etanol). Las mezclas se sometieron a irradiación con microondas $\left(\right.$ a $80^{\circ} \mathrm{C}$; rampa de calentamiento: $5^{\circ} \mathrm{C} \cdot \mathrm{min}^{-1}$ ) y a agitación durante 20 minutos en un horno microondas Ethos-One (Milestone, Sorisole, BG, Italia). Los productos resultantes se aislaron por centrifugación (2500 rpm) y se almacenaron a $4{ }^{\circ} \mathrm{C}$. 


\section{CONGRESO IBÉRICO DE AGROINGENIERÍA \\ X CONGRESSO IBÉRICO DE AGROENGENHARIA \\ 3 - 6 septiembre 2019, Huesca - España}

Los composites se caracterizaron mediante espectroscopia infrarroja de transformada de Fourier, microscopía electrónica de barrido y microscopía electrónica de transmisión, con resultados de caracterización coherentes con los descritos en la patente P201731489 [8].

\subsection{Aislado fúngico y condiciones de crecimiento}

El aislado MYC43 de Phytophthora cinnamomi fue suministrado por el Centro de Investigaciones Científicas y Tecnológicas de Extremadura-Instituto del Corcho, Madera y Carbón Vegetal, España. El aislado se mantuvo en tubos con medio patata-dextrosa-agar (PDA), suministrados por Merck Millipore (Darmstadt, Alemania), almacenados a $4{ }^{\circ} \mathrm{C}$. Se realizaron nuevos subcultivos para obtener el inóculo para las pruebas de sensibilidad in vitro transfiriendo tapones de hifas a placas de Petri con PDA como medio.

\subsection{Pruebas in vitro de inhibición del crecimiento micelial}

La actividad biológica de los materiales compuestos se determinó mediante el método de dilución en agar: se incorporaron alícuotas de las soluciones madre de los diversos tratamientos en el medio de PDA para obtener concentraciones finales de 125, 250 y $500 \mu \mathrm{g} \cdot \mathrm{mL}^{-1}$. Los discos miceliales de patógeno ( $8 \mathrm{~mm}$ de diámetro), retirados de los márgenes de un cultivo de 7 días, se transfirieron luego a las placas de PDA, empleando placas que contenían solo el medio de PDA como control. Se realizaron tres réplicas por tratamiento.

La inhibición del crecimiento micelial (o la eficacia del compuesto analizado) para cada tratamiento y concentración después de 7 días de incubación, en la oscuridad, se calculó de acuerdo con la fórmula: $\left(\left(d_{c}-d_{t}\right) / d_{c}\right) \times 100$, donde $d_{c}$ corresponde al diámetro promedio de la colonia de hongos en el control y $d_{\mathrm{t}}$ al diámetro promedio de la colonia de hongos tratada con el compuesto ensayado [9].

Los resultados se expresaron también como concentraciones efectivas CE50 y CE90 (es decir, aquellas concentraciones que redujeron el crecimiento del micelio en un $50 \%$ y un $90 \%$, respectivamente), que se determinaron mediante la regresión de los valores de inhibición del crecimiento radial (\%) frente a los valores $\log _{10}$ de las concentraciones de los compuestos antifúngicos.

\subsection{Análisis estadístico}

Los datos de los resultados obtenidos en este documento y de los informados en un estudio previo con AgNPs [7] se sometieron a análisis de varianza (ANOVA). Para la comparación post hoc de las medias se utilizó el test de Tukey con un nivel de probabilidad de 0,05 ( $p<0,05$ ). Todos los análisis se realizaron con el software IBM SPSS Statistics v.25.

\section{Resultados y discusión}

La bioactividad de los distintos tratamientos se estudió in vitro controlando el crecimiento radial del micelio (Figura 1).

Los resultados de crecimiento radial del micelio se muestran en la Figura 2, comparando los tratamientos sin AgNPs con los tratamientos con AgNPs recogidos en la referencia [7]. El aumento de la concentración de los complejos de inclusión de $125 \mu \mathrm{g} \cdot \mathrm{mL}^{-1}$ a $500 \mu \mathrm{g} \cdot \mathrm{mL}^{-1}$ condujo a una reducción del crecimiento micelial en todos los casos.

Se puede observar que la adición de AgNPs sólo mejoró de forma notable la actividad de los compuestos a base de ácido gálico. Para los compuestos a base de ácido ferúlico, la mejora fue estadísticamente significativa sólo en la dosis más baja; y para los compuestos basados en curcumina o silimarina, la adición de AgNPs llevó a un peor comportamiento (especialmente evidente para el compuesto con silimarina a la concentración más alta de $\left.500 \mu \mathrm{g} \cdot \mathrm{mL}^{-1}\right)$. 
Los resultados del ANOVA factorial (Tabla 1) indicaron efectos de interacción unidireccionales, bidireccionales y triples estadísticamente significativos. En la Tabla 2 se muestra una clasificación de los tratamientos, con y sin AgNPs, según el test de Tukey.

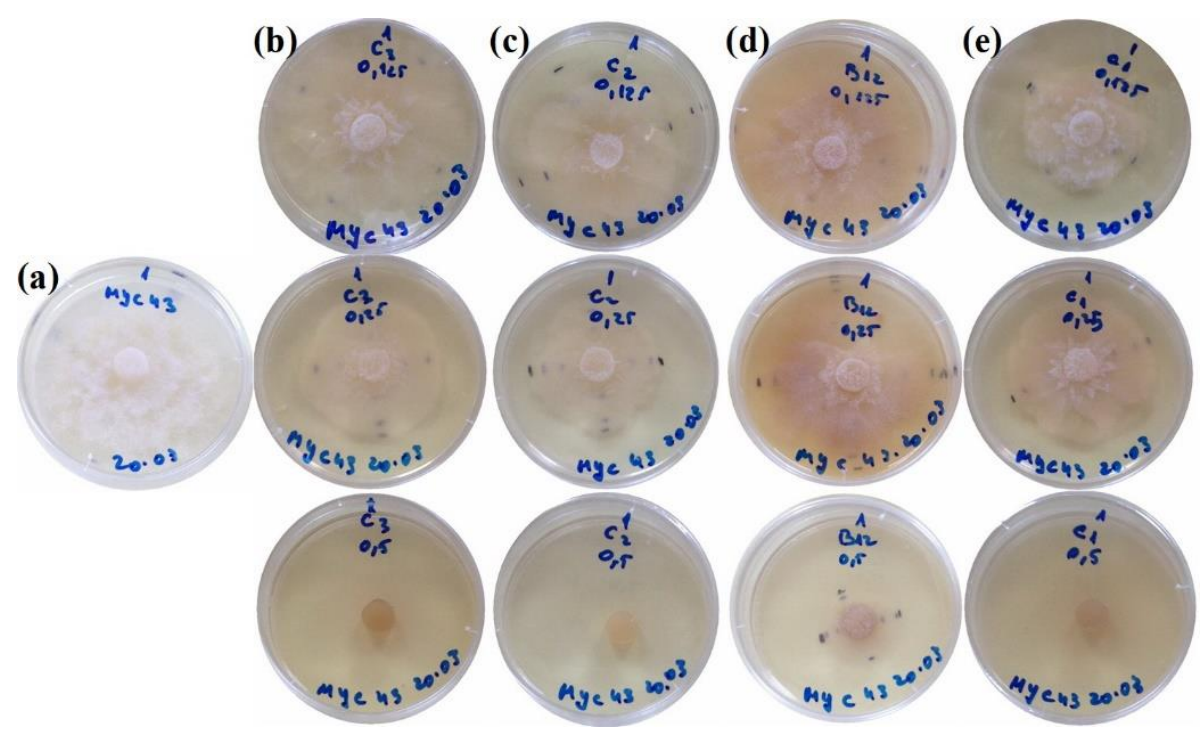

Figura 1. Ejemplo de test de sensibilidad. Crecimiento radial del micelio para (a) el control, y tratamientos con (b) curcumina, (c) ácido ferúlico, (d) ácido gálico y (e) silimarina. De arriba abajo: concentraciones de $125 \mu \mathrm{g} \cdot \mathrm{mL}^{-1}, 250 \mu \mathrm{g} \cdot \mathrm{mL}^{-1}$ y $500 \mu \mathrm{g} \cdot \mathrm{mL}^{-1}$. Sólo se muestra una repetición por tratamiento.

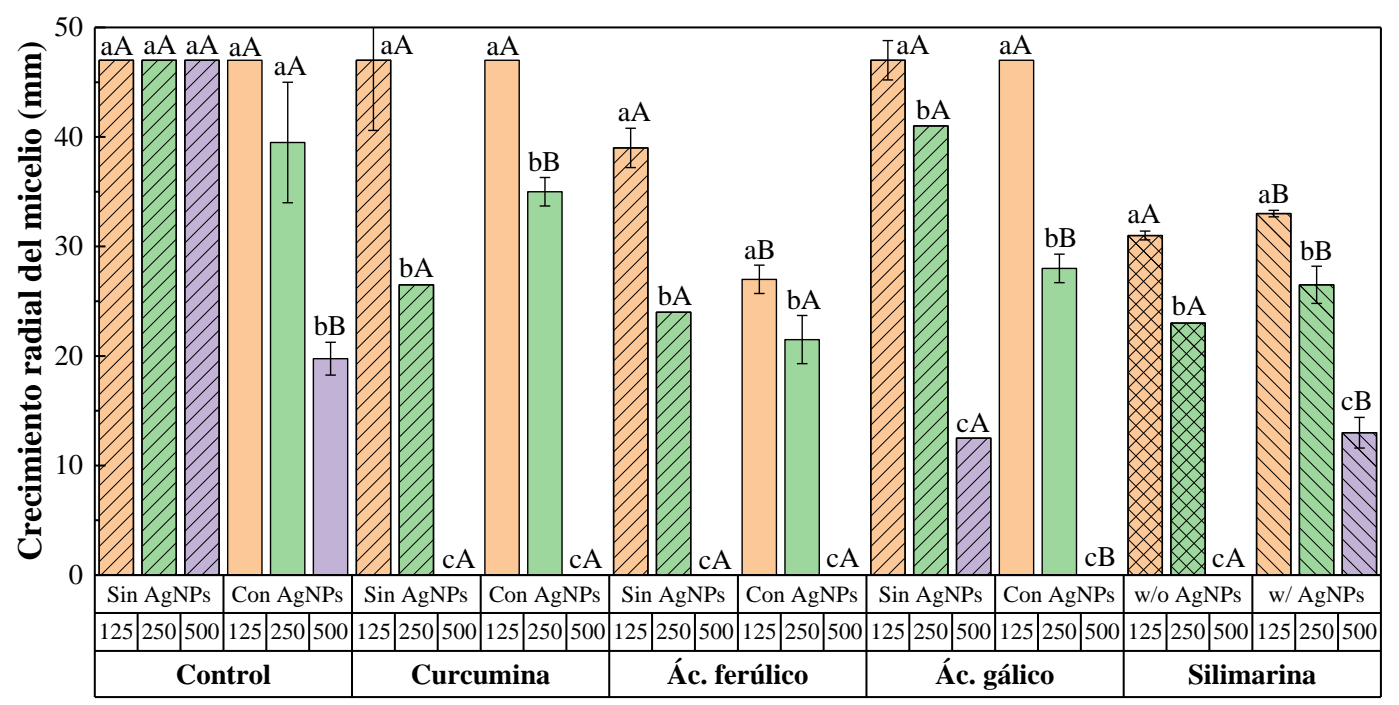

Figura 2. Valores de crecimiento radial de $P$. cinnamomi en presencia de los composites, consistentes en complejos de inclusión de polifenoles sin y con nanopartículas de plata (AgNPs), a diferentes concentraciones (expresadas en $\mu \mathrm{g} \cdot \mathrm{mL}^{-1}$ ). Para cada tratamiento, las concentraciones etiquetadas con la misma letra minúscula no son estadísticamente diferentes según el test de Tukey $(p<0,05)$. Para los tratamientos con el mismo polifenol y la misma dosis, las letras mayúsculas diferentes indican que la ausencia/presencia de AgNPs dió lugar a diferencias significativas según el test de Tukey $(p<0,05)$. Todos los valores presentados son valores medios de tres repeticiones. Las barras de error representan las desviaciones estándar de tres repeticiones. 
X CONGRESO IBÉRICO DE AGROINGENIERÍA

X CONGRESSO IBÉRICO DE AGROENGENHARIA

3 - 6 septiembre 2019, Huesca - España

Tabla 1. Test de efectos entre sujetos.

\begin{tabular}{cccccc}
\hline Fuente & GL & Suma de cuadrados & Cuadrados medios & F & Pr $>$ F \\
\hline Tratamiento & 4 & 5647,375 & 1411,844 & 446,080 & $<0,0001$ \\
Dosis & 2 & 16053,013 & 8026,506 & 2536,021 & $<0,0001$ \\
AgNPs & 1 & 228,006 & 228,006 & 72,040 & $<0,0001$ \\
Tratamiento*Dosis & 8 & 2103,425 & 262,928 & 83,074 & $<0,0001$ \\
Tratamiento*AgNPs & 4 & 1013,275 & 253,319 & 80,038 & $<0,0001$ \\
Dosis*AgNPs & 2 & 52,962 & 26,481 & 8,367 & 0,001 \\
Tratamiento*Dosis*AgNPs & 8 & 1003,475 & 125,434 & 39,632 & $<0,0001$ \\
\hline
\end{tabular}

GL: grados de libertad

Tabla 2. Categorías en función de los valores de crecimiento radial del micelio para cada combinación de polifenol*dosis*AgNPs, con un intervalo de confianza del 95\%, según el test de Tukey.

\begin{tabular}{|c|c|c|c|c|c|c|c|c|c|c|c|}
\hline Categoría & & & & & & rupo & & & & & \\
\hline Ác. ferúlico*500*con AgNPs & A & & & & & & & & & & \\
\hline Curcumina ${ }^{*} 500^{*} \sin$ AgNPs & A & & & & & & & & & & \\
\hline Curcumina* $500^{*}$ con AgNPs & A & & & & & & & & & & \\
\hline Ác. ferúlico* $500^{*} \sin$ AgNPs & A & & & & & & & & & & \\
\hline Ác. gálico*500*con AgNPs & A & & & & & & & & & & \\
\hline Silimarina*500*sin AgNPs & A & & & & & & & & & & \\
\hline Ác. gálico*500*sin AgNPs & & B & & & & & & & & & \\
\hline Silimarina ${ }^{*} 500^{*}$ con AgNPs & & B & & & & & & & & & \\
\hline Control ${ }^{*} 500^{*}$ con AgNPs & & & $\mathrm{C}$ & & & & & & & & \\
\hline Ác. ferúlico $250^{*}$ con AgNPs & & & $\mathrm{C}$ & $\mathrm{D}$ & & & & & & & \\
\hline Silimarina*250*sin AgNPs & & & $\mathrm{C}$ & $\mathrm{D}$ & E & & & & & & \\
\hline Ác. ferúlico*250* sin AgNPs & & & $\mathrm{C}$ & $\mathrm{D}$ & E & & & & & & \\
\hline Silimarina ${ }^{*} 250^{*}$ con AgNPs & & & & $\mathrm{D}$ & E & $\mathrm{F}$ & & & & & \\
\hline Curcumina*250*sin AgNPs & & & & $\mathrm{D}$ & E & $\mathrm{F}$ & & & & & \\
\hline Ác. ferúlico ${ }^{*} 125^{*}$ con AgNPs & & & & $\mathrm{D}$ & E & $\mathrm{F}$ & & & & & \\
\hline Ác. gálico*250*con AgNPs & & & & & E & $\mathrm{F}$ & G & & & & \\
\hline Silimarina* $125^{*} \sin$ AgNPs & & & & & & $\mathrm{F}$ & G & $\mathrm{H}$ & & & \\
\hline Silimarina ${ }^{*} 125^{*}$ con AgNPs & & & & & & & G & $\mathrm{H}$ & & & \\
\hline Curcumina* $250^{*}$ con AgNPs & & & & & & & & $\mathrm{H}$ & I & & \\
\hline Ác. ferúlico ${ }^{*} 125^{*} \sin$ AgNPs & & & & & & & & & I & $\mathrm{J}$ & \\
\hline Control ${ }^{*} 250^{*}$ con AgNPs & & & & & & & & & I & $\mathrm{J}$ & \\
\hline Ác. gálico*250*sin AgNPs & & & & & & & & & & $\mathrm{J}$ & \\
\hline Control ${ }^{*} 250 * \sin$ AgNPs & & & & & & & & & & & K \\
\hline Control $^{*} 125^{*}$ con AgNPs & & & & & & & & & & & K \\
\hline Control $^{*} 125^{*} \sin$ AgNPs & & & & & & & & & & & K \\
\hline Curcumina* $125^{*}$ con AgNPs & & & & & & & & & & & K \\
\hline Curcumina ${ }^{*} 125^{*} \sin$ AgNPs & & & & & & & & & & & K \\
\hline Ác. gálico* $125^{*}$ con AgNPs & & & & & & & & & & & K \\
\hline Ác. gálico* $125^{*} \sin$ AgNPs & & & & & & & & & & & K \\
\hline Control*500*sin AgNPs & & & & & & & & & & & $\mathrm{K}$ \\
\hline
\end{tabular}

Valor crítico $d$ de Tukey: 5,566

Los resultados del test de sensibilidad también pueden expresarse en términos de indicadores de concentración efectiva CE50 y CE90. La sensibilidad de los aislados claramente varió en función de la ausencia/presencia de AgNPs en los composites y dependiendo del compuesto fenólico empleado. La adición de AgNPs apenas modificó los valores de CE90 para curcumina y 


\section{CONGRESO IBÉRICO DE AGROINGENIERÍA \\ X CONGRESSO IBÉRICO DE AGROENGENHARIA \\ 3 - 6 septiembre 2019, Huesca - España}

ácido ferúlico (los dos polifenoles con mejor actividad antifúngica), pero mejoró notablemente los de los composites de ácido gálico (en un 34\% y 112\% para CE50 y CE90, respectivamente).

Tabla 3. Concentraciones efectivas que inhibieron el crecimiento micelial en un $50 \%$ y un $90 \%$ (CE50 y CE90, respectivamente).

\begin{tabular}{ccccc}
\hline \multirow{2}{*}{ Tratamiento } & \multicolumn{2}{c}{$\mathrm{CE}_{50}\left(\mu \mathrm{g} \cdot \mathrm{mL}^{-1}\right)$} & \multicolumn{2}{c}{$\mathrm{CE}_{\mathbf{9 0}}\left(\mu \mathrm{g} \cdot \mathrm{mL}^{-1}\right)$} \\
\cline { 2 - 5 } & Sin AgNPs & Con AgNPs & Sin AgNPs & Con AgNPs \\
\hline Control & -- & 458,4 & -- & 1192,8 \\
Curcumina & 257,5 & 279,9 & 448,3 & 487,4 \\
Ác. ferúlico & 228,7 & 171,6 & 446,2 & 450,4 \\
Ác. gálico & 373,6 & 261,3 & 795,3 & 455,6 \\
Silimarina & 195,5 & 261,8 & 453,1 & 963,7 \\
\hline
\end{tabular}

Con respecto al impacto de la elección del polifenol, para los tratamientos sin AgNPs, tanto en base a la Tabla 2 como a los valores de CE50, la eficacia seguiría la secuencia: silimarina > ácido ferúlico > curcumina > ácido gálico. En base a los valores de $C_{90}$, las sensibilidades de $P$. cinnamomi a la curcumina, el ácido ferúlico y la silimarina serían similares, y el ácido gálico sería la opción menos deseable.

De acuerdo con el método de Wadley [10] para la cuantificación del nivel de interacción, se obtuvo un factor de sinergia $\mathrm{FS}=1,7$ para el ácido gálico, indicativo de una interacción sinérgica con las AgNPs; para el ácido ferúlico FS=1,0, indicativo de un comportamiento aditivo entre los dos productos antifúngicos; y para silimarina y curcumina se obtuvieron valores de FS de $0,5 \mathrm{y}$ 0,9 , respectivamente, indicativos de un comportamiento antagónico con la nanoplata.

Aunque poco frecuentes en la literatura, es preciso aclarar que, por ejemplo, se han referido casos de comportamiento antagónico de las AgNPs con amoxicilina frente a Staphylococcus aureus resistente a meticilina (SARM) [11]. Estos autores, en ensayos con 7 organismos y 19 antibióticos, completaron 96 tests, encontrando 5 combinaciones con comportamiento sinérgico, 89 con comportamiento aditivo y 2 con comportamiento antagonístico.

\section{Conclusiones}

Los ensayos realizados han conducido a diferencias notables en la inhibición del crecimiento del micelio de P. cinnamomi, observándose un rendimiento superior de los preparados a base de ácido ferúlico con AgNPs y de silimarina sin nAg (evidenciando, así, que la adición de AgNPs, pese a su actividad antimicrobiana, no siempre da lugar a sinergismos).

Los principales hallazgos han sido: un comportamiento antagonístico inesperado para dos de los polifenoles (curcumina y silimarina), un comportamiento aditivo para el ácido ferúlico y un comportamiento sinergístico para el ácido gálico.

La conclusión alcanzada es que los preparados referidos pueden ser prometedores para las aplicaciones de protección de cultivos y masas forestales contra P. cinnamomi.

\section{Agradecimientos}

Esta investigación ha sido financiada a través del proyecto VA258P18 de la Junta de Castilla y León. 


\section{CONGRESO IBÉRICO DE AGROINGENIERÍA \\ X CONGRESSO IBÉRICO DE AGROENGENHARIA \\ 3 - 6 septiembre 2019, Huesca - España}

\section{Referencias}

1. Ramírez Gil, J.G. Incidencia, diagnóstico, comportamiento y alternativas de manejo de la marchitez del aguacate con énfasis en Phytophthora cinnamomi Rands. Universidad Nacional de Colombia, Medellín, Colombia, 2013.

2. Bergot, M.; Cloppet, E.; Perarnaud, V.; Deque, M.; Marcais, B.; Desprez-Loustau, M.-L. Simulation of potential range expansion of oak disease caused by Phytophthora cinnamomi under climate change. Global Change Biol. 2004, 10, 1539-1552.

3. Lowe, S.; Browne, M.; Boudjelas, S.; De Poorter, M. 100 of the world's worst invasive alien species: a selection from the global invasive species database. Invasive Species Specialist Group Auckland: Auckland, NZ, 2004; $\mathrm{p} 12$.

4. Sánchez, M.; Caetano, P.; Romero, M.; Navarro, R.; Trapero, A. Phytophthora root rot as the main factor of oak decline in southern Spain. In Progress in research on Phytophthora diseases of forest trees, Brasier, C.; Jung, T.; Oßwald, W., Eds. Forest Research: Farnham, Surrey, UK, 2004; pp 149-154.

5. Jackson, T.J.; Burgess, T.; Colquhoun, I.; Hardy, G.E.S. Action of the fungicide phosphite on Eucalyptus marginata inoculated with Phytophthora cinnamomi. Plant Pathol. 2000, 49, 147-154.

6. Sun, T.; Zhou, D.; Xie, J.; Mao, F. Preparation of chitosan oligomers and their antioxidant activity. Eur. Food Res. Technol. 2007, 225, 451-456.

7. Matei, P.; Martín-Gil, J.; Michaela Iacomi, B.; Pérez-Lebeña, E.; Barrio-Arredondo, M.; Martín-Ramos, P. Silver nanoparticles and polyphenol inclusion compounds composites for Phytophthora cinnamomi mycelial growth inhibition. Antibiotics 2018, 7.

8. Martín-Gil, J.; Matei Petruta, M.; Pérez Lebeña, E. P201731489, Complejo de inclusión para mejorar la biodisponibilidad de compuestos biológicamente activos no hidrosolubles. 28th December 2017, 2017.

9. Şesan, T.E.; Enache, E.; Iacomi, B.M.; Oprea, M.; Oancea, F.; Iacomi, C. In vitro antifungal activity of some plant extracts against Fusarium oxysporum in blackcurrant (Ribes nigrum L.). Acta Sci. Pol. Hortorum 2017, 16, 167-176.

10. Wadley, F.M. The evidence required to show synergistic action of insecticides and a short cut in analysis; U.S. Government Printing Office: Washington DC, USA, 1945.

11. De Souza, A.; Mehta, D.; Leavitt, R. Bactericidal activity of combinations of Silver-Water Dispersion with 19 antibiotics against seven microbial strains. Curr. Sci. 2006, 91, 1825-1851. 